\title{
Ground Sediment Transport Model and Numerical Simulation
}

\author{
Jichao Sun ${ }^{1,2 *}$ \\ ${ }^{1}$ School of Water Resources and the Environment, China University of Geosciences, \\ Beijing, 100083, China \\ ${ }^{2}$ Department of Civil \& Environmental Engineering, University of Wisconsin, \\ Milwaukee 53211, United States
}

Received: 22 September 2014

Accepted: 26 November 2015

\begin{abstract}
Geological disasters caused by city ground subsidence are typical examples of soil failure, which significantly impact urban planning and development. The sediment transport in the soils leads to the increasing porosity of soils, and changes the elastic modulus and Poisson's ratio, thus the carrying capacity of the soil is reduced and ground subsidence appears. Therefore, the sediment transfer and loss driven by the underground water becomes a key reason for ground subsidence. Studies in this area thus present significant meanings. In this paper, mutual effects between the sediment particles and groundwater seepage are analyzed. We partially fix sediments in soils. Using the numerical simulation, hydraulic pressure is applied to scour so as to study the regular patterns of sediment transport in the soil mass: as time extended, the porosity in soils is gradually enlarged, tending to be stable; as more particles are fixed, porosity is reduced. It is also found that fixed particles, with a part of free sediment particles, form some new fixed sediment structures that hinder the transfer of the free sediment. In addition, parameters like scour time, hydraulic pressure, and the porosity evolution equation relating to the amount of fixed sediment particles are acquired. Findings of this research are expected to provide references for predicting, forecasting, and treating ground subsidence, which is led by sediment transport from the perspective of the soil mass porosity evolution.
\end{abstract}

Keywords: ground sediment, water and sediment interaction, soils collapse

\section{Introduction}

A wide range of ground subsidence disasters have occurred all around the world. The most representative example - regarded a super large ground subsidence incidence - was a huge crater about $61 \mathrm{~m}$ deep in Guatemala City street in 2010. Ground subsidence

*e-mail: Jichao@email.com Jichao@uwm.edu disasters have caused numerous losses of life and property, and significantly impacted city planning and development. Riverbank collapses also have occurred frequently [1-3]. All of this has made the interaction between sediment and water that leads to soil collapse a hot research topic [1-3].

Currently, views on ground subsidence can be divided into two aspects: karst collapse and the extraction of groundwater. Both views can explain the ground subsidence phenomenon effectively, but there are still many cases that cannot be explained, such as the tilting, subsidence, and damage to surrounding buildings caused 
by pit excavation of a high-rise building foundation and subway excavation and pumping. In order to analyze these phenomena, a new interpretation is proposed in this study: that underground sediment transport and increased porosity generated the soil mass damage. The transfer and loss of sediment in soil mass increases soil mass porosity, leading to the overall decline of elastic modulus of soils, enhancing the deformation, reducing the carrying capacity, and leading to the settlement of the ground even the overall collapse of the ground.

Many previous studies about sediment are mainly about surface sediment transport $[2,3]$. A new research direction is the underground sediment, which is the sediment inside the soils. The research in this paper is about ground sediment scouring research, the concept and model of which are set up in the literature [4-9].

\section{Current Research Conditions in this Field}

There is a lot of work about the transport of colloids, the entrapment of those particles by porous media grains, and the re-entrainment of particles - including the transport of engineered nanoparticles in soils [7], the behavior of foul types of untreated metal oxide nanoparticles in saturated porous media $[8,9]$, and the contaminant-colloidal fines associations and the colloidal fines release mobilization, entrapment, or plugging and migration processes [10-14]. But this paper studies the sediment particles in soils.

In this study the main discipline is the field of fluid mechanics, mainly as the water seepage in saturated [15] and unsaturated soils [16, 17]. With the developing studies of sediment transport, the definition of the state points was discussed based on the experimental and numerical calculation [16-18]. The studies on related theories of sediment transfer in the underground water - especially on the quantization transport process - are currently insufficient. These researchers obtained the critical hydraulic grade, only by experiments, while they lack theoretical analysis and the mathematical derivation.

As for the experimental studies, two processes have been observed, namely subsurface erosion and volume swelling [19], and it has been drawn from model experiments that the irreversible displacement of each soil particle corresponds to a critical hydraulic gradient [20]. Moreover, relationships of some parameters were clarified. For example, Sentko came to the conclusion that the time of sand loosening from the beginning to the end is positively proportional to the thickness of the base, and negatively proportional to the permeability coefficient of Darcy [21]; in addition, influencing factors such as pressure head, fiber content, fiber length, and so on under different combinations of soil and fibers were analyzed [22]. Piping conditions and the blocking effects of soils on piping were obtained by making experiments [22-24]. These findings of experiments presented certain values of field applications that can be used to help avoid piping in the field. Yet all this research is still short of studies on the porosity evolution regularities of the soil mass under conditions lacking scouring effects. That is, the evolution process of porosity during scouring is not concerned.

As for the theoretical analysis, the stress on particles in the pore channel was analyzed, and the critical flow velocity formula during piping was obtained [25]. However, sediment particles [25] were sands without viscosity, and particles had the same particle size. Actually, soils may be formed by the combination of cohesive soil and non-cohesive soils or sandwiched sands [26]. Thus, the research result may not be consistent with the actual situation. Thus, the application shows certain limitations.

As for numerical computation and numerical simulation, many scientists conducted the numerical simulation on the piping of Jinjiang River Dam under different conditions, including simulation of seepage velocities under different flood peaks and different seepage coefficients [27]. Flac3D was used to study soil pipes in the soil slope, reaching the conclusion that water flow in soil tube and the evolution of soil tube influenced the stability of the slope soils [28]. However, the transfer of sediment particles in tubes is neglected [27, 28].

In this paper, the study focused on the analysis of increasing porosity in soils under the condition of the scour and loss of sediment particles in soils by seepage water; in addition, the mutual effects between soil particles and water are analyzed, that is, the coupling between the solid and fluid particles so as to acquire the evolution of the soil mass porosity.

\section{Theoretical Basis}

Mutual effects between soil particles and water belong to the fluid-structure interaction, with the connection worked through a force. Therefore, in the analysis the mass conservation of fluid and solid - as well as the coupling force between the fluid and solid - should be obtained. Thus the calculation should be carried out on the premise of the analysis of discrete elements.

In discrete elements, compared with the deformation of the pore volume among the solid particles, the water unit can be assumed as incompressible fluid with constant density. Each calculation step shall meet the NavierStokes continuity and kinematic equations [29] as follows:

$$
\frac{\partial n}{\partial t}=-(\nabla \cdot n u)
$$

$$
\frac{\partial(n u)}{\partial t}=-(\nabla \cdot n u u)-\frac{n}{\rho_{f}} \nabla p-\frac{n}{\rho_{f}} \nabla \cdot \tau+n g+\frac{f_{\text {int }}}{\rho_{f}}
$$

...where $u$ is the velocity vector, $t$ is the viscous stress tensor, $g$ is acceleration due to gravity, $n$ is the porosity $t$ is time, $\nabla$ is the gradient and $\nabla=\{\partial / \partial \mathrm{x}, \partial / \partial \mathrm{y}, \partial / \partial \mathrm{z}\}$, $\nabla p$ is fluid pressure gradient, $\rho_{\mathrm{f}}$ is fluid density, and $f_{\text {int }}$ is the mutual force between fluid and solid particles in unit volume (including the drag between fluid and solid 
particles). This can be obtained by fluid pressure gradient equation. The general expression is as follows:

$$
f_{\text {int } f}=n \nabla p_{j} \text {, that is } f_{\text {int } f}=\beta_{\text {int } j}\left(v_{j}-u_{j}\right)
$$

...where $\nabla p_{j}$ is the fluid pressure gradient component, $u_{j}$ is average flow velocity, and $v_{j}$ is the average velocity of sand particles.

Eq. (1) can be unfolded as:

$$
\frac{\partial n}{\partial t}+\frac{\partial\left(n u_{x}\right)}{\partial x}+\frac{\partial\left(n u_{y}\right)}{\partial y}+\frac{\partial\left(n u_{z}\right)}{\partial z}=0
$$

Eq. (3) can be changed into [30-32],

$$
\nabla p=\left(150 \frac{(1-n)^{2}}{n^{2} \bar{d}_{p}^{2}} \rho_{f} \mu_{f}+1.75 \frac{(1-n) \rho_{f}}{n \bar{d}_{p}}\left|v_{j}-u_{f}\right|\right) \cdot\left(v_{j}-u_{f}\right)
$$

...where $\mu_{\mathrm{f}}$ is the hysteresis coefficient, $\bar{d}_{p}$ is the average particle size, $u_{j}$ is the average flow velocity, and $v_{j}$ is the average velocity of sand particles.

\section{Transfer of Underground Sediments in Soils}

The soil mass is formed by substantial fine particles Fine particles are bonded to each other to further constitute larger particles, and ultimately the formation of the soil mass. As particles cannot be fully dense, the presence of pores in the soil can be observed. In pores, some small particles are in the dispersion state. This part of sediment particles is transferred under the vadose water hydro dynamism. With the enlarged water flow velocity, the adhesive strength among the originally bonded soil particles is damaged. The soil particles without adhesive strength are also transferred by the hydraulic drive.

The transfer of sediment particles in the soil driven by the water will be imposed to the following mechanical effects: the hydraulic effect, collision with mobile sediments in water, and friction from fixed sediment particles of soils in the wall of the vadose passage [5]. With complex and diverse forms of forces, it is difficult to interpret the process by theoretical analysis or analytical solutions. Hence in this study, the transport model of sediment particles is established, including the simple transfer model of sediment particles and multiple transfer model of sediment particles transfer.

\section{Transfer of Single Particles through the Large Sediment Particles}

In the soil mass, diameters of sediment particles vary. Under the action of the hydraulic transfer, sediment particles with smaller size are more prone to be transported and the sediment particles of larger sizes are hard to move. The soil mass is a three-dimensional spatial structure but the study based on three-dimensional spatial structure is extremely complex. Hence, in this research, the twodimensional approach is adopted.

In the soil mass, sediment particles are bound to constraints from three dimensions and six directions (up, down, front, back, left, and right). However, in the twodimensional plane only four directions can be studied (up, down, left, and right). Large sediment particles can be easily constrained and one pair of the key constraining directions is front and back. Therefore, it is a smart solution to fix large particles in the two-dimensional research of underground sediment particles.

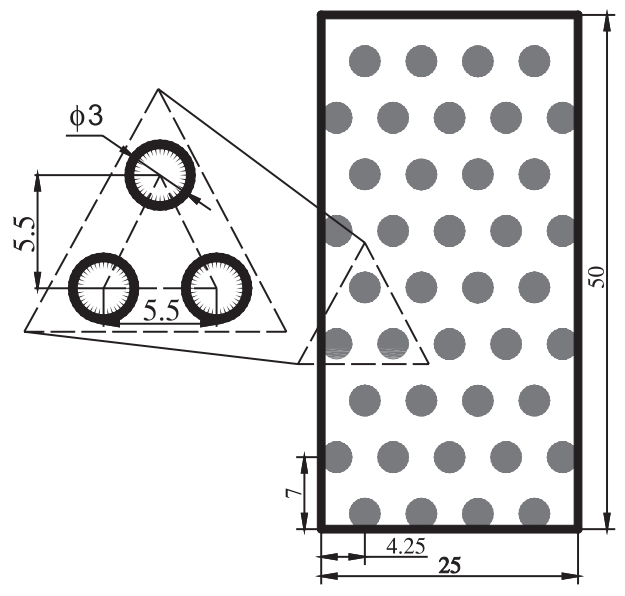

A
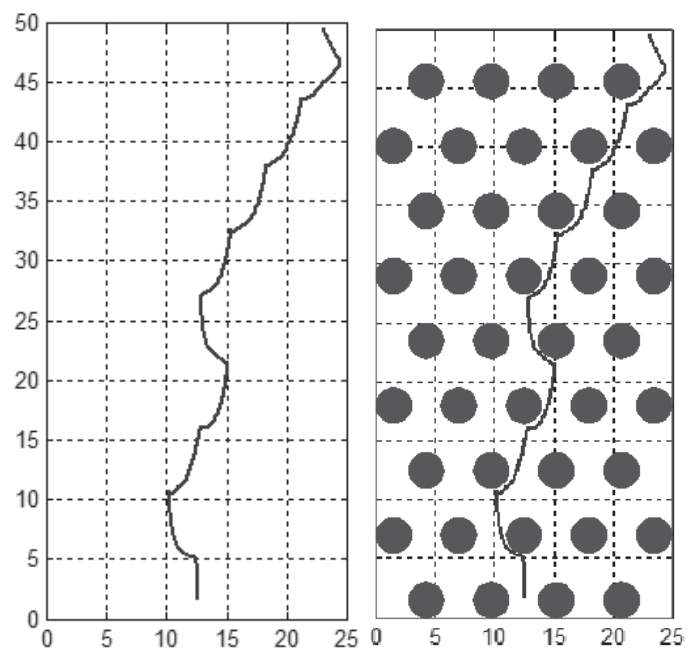

B

Fig. 1. Spatial distribution and track of sediment particles.

A - Spatial distribution of sediments (the unit is $\mathrm{cm}$ ), B - Track of central point of sediment particles. 


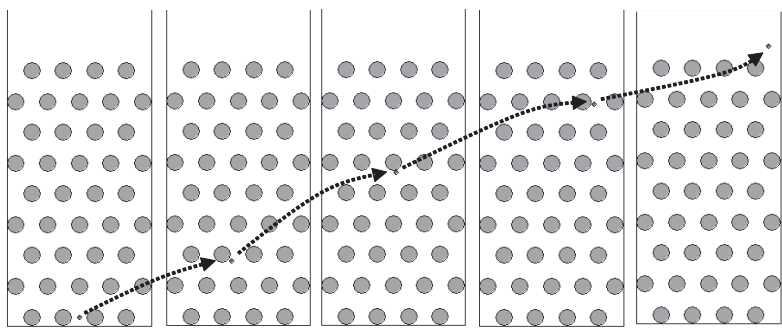

Fig. 2. Position of single particle at $0,0.5,1,1.5$, and 2 seconds.

A numerical model is built up for this condition for numerical calculation. Specific parameters of the numerical model are as follows: the diameter of a large sediment particle is about $0.03 \mathrm{~m}$, the diameter of a single sediment particle is $0.0075 \mathrm{~m}$, the research area is $0.25 \mathrm{~m}$ wide and $0.5 \mathrm{~m}$ high, the horizontal spacing of large particles is $0.055 \mathrm{~m}$, and the vertical spacing of large particles is $0.055 \mathrm{~m}$. As shown in Fig. 1A, the unit of numbers in the figure is $\mathrm{cm}$.

The mechanical parameters include particle density of $1,500 \mathrm{~kg} / \mathrm{m}^{3}$, the elastic modulus of the particles is $1,000 \mathrm{Kpa}$, the friction coefficient is 0.5 , the elastic modulus of surrounding walls is $1,000 \mathrm{Kpa}$, and the friction coefficient is 0.3 ; the bottom hydraulic pressure is $55 \mathrm{KPa}$. The result is shown in Figs $1 \mathrm{~B}$ and 2.

The following phenomenon is found in the numerical simulation: the free sediment particles are scoured by water and moved along the water flow. In the movement, free sediment particles collide with larger particles, perhaps several times. The collision changes the movement velocity direction of mobile sediment particles; the particles are transferred and advanced in the fractures formed by fixed sediment particles.

It can be observed from the advancing track of small sediment particles that under the force of water flow, the movement of small particles sediment is basically consistent with the direction of water flow. When small particles and large particles collide, friction changes the movement direction of small particles.

\section{Transfer of Underground Sediment Particles in Soil Mass}

\section{Scour Simulation}

Sediment particles of three different sizes have radii of $0.00375 \mathrm{~m}, 0.003 \mathrm{~m}, 0.002 \mathrm{~m}$, and $0.001 \mathrm{~m}$, respectively, corresponding to the amounts of 700,900, 1,500 and 2,100. So a total of 5,200 particles are involved. The size of the soil mass is $0.25 \mathrm{~m} \times 0.5 \mathrm{~m}$, and the hydraulic pressure is selected as $10 \mathrm{KPa}, 20 \mathrm{KPa}$, and $30 \mathrm{KPa}$. The upward hydraulic pressure is applied on the bottom of the soil mass. The applied time is instant and the scouring time is $1 \mathrm{~s}$. We randomly fixed $600(11.54 \%), 800(15.38 \%)$, and $1,000(19.23 \%)$ sediment particles to form the random soil mass of fixed particles as shown in Fig. 3.

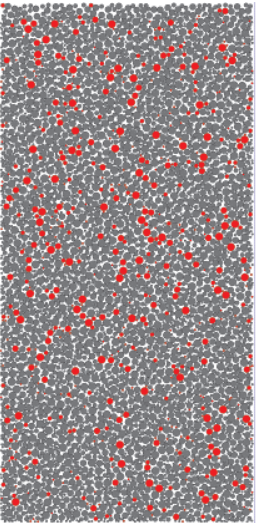

$11.54 \%$

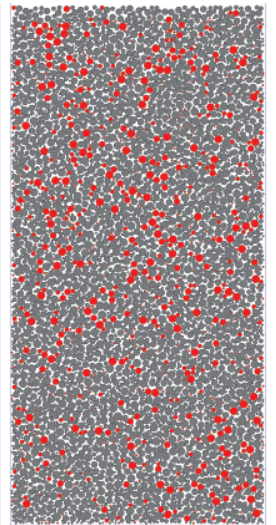

$15.38 \%$

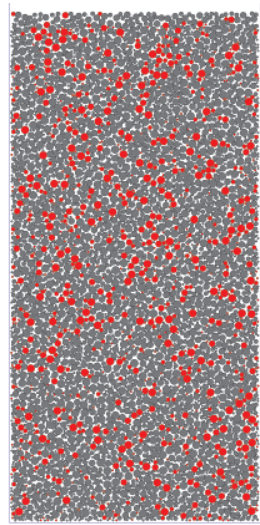

$19.23 \%$
Fig. 3. Spatial distributions of fixed particles (red as fixed particles, grey as free particles).

Upward hydraulic pressure is applied on the bottom of the soil mass.

And the scouring started and continued. The distributions of sediment particles at different times are shown in Fig. 4, which shows the spatial distribution of fixed sediments and free sediments of $11.54 \%$ fixed sediments at $0.2 \mathrm{~s}(\mathrm{~A}), 0.6 \mathrm{~s}(\mathrm{~B})$, and $1.0 \mathrm{~s}(\mathrm{C})$.

Throughout the calculation, the monitoring results of the porosity of sediment particles with fixations of $11.54 \%, 15.38 \%$, and $19.23 \%$ with an interval of $0.1 \mathrm{~s}$ and duration of $1 \mathrm{~s}$ are obtained as shown in Figs $6 \mathrm{~A}, \mathrm{~B}$, and C.

The simulation process can be described as follows:

1. Non-fixed sediment particles are transferred under the scouring action of the water flow. Particles have a mutual extrusion force (shown in Fig. 7B). The bottom free particles are influenced by the water flow and moved upwards, pushing away the front and surrounding sediment particles.

2. In the upward movement, when encountering fixed particles, free particles are blocked by fixed particles.

3. In the transfer of sediment particles, fracture-caves and pores are formed. A very small amount of sediment

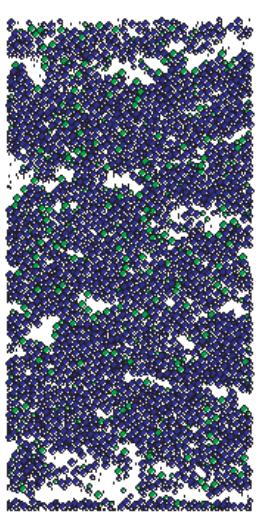

(A) $0.2 \mathrm{~s}$

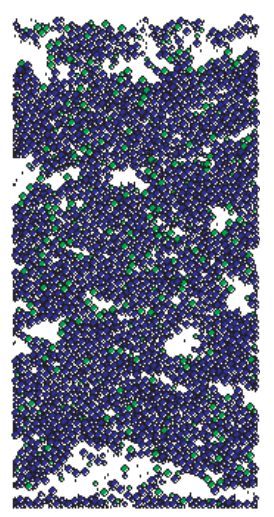

(B) $0.6 \mathrm{~s}$

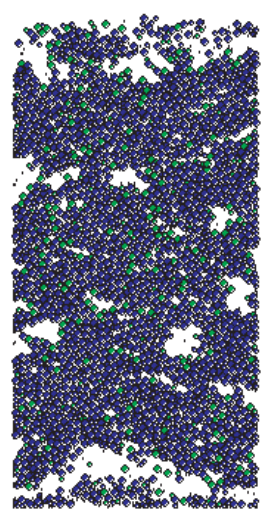

(C) $1.0 \mathrm{~s}$
Fig. 4. Porosity evolution of the soil mass with $11.54 \%$ fixed particles: blue as free particles, green as fixed particles. 
particles have advance and return movement in fracture-caves and pores to a certain extent.

The following regularities can be seen from Figs 6A, $\mathrm{B}$, and $\mathrm{C}$ :

1. As time is extended, porosity is gradually increased and the increased range is gradually decreased; in addition, the larger the amount of fixed particles, the

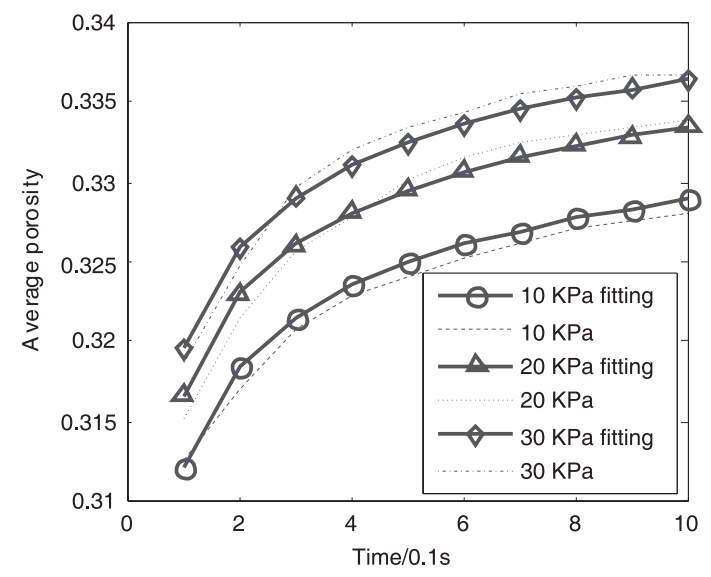

(A) $11.54 \%$ particles fixed

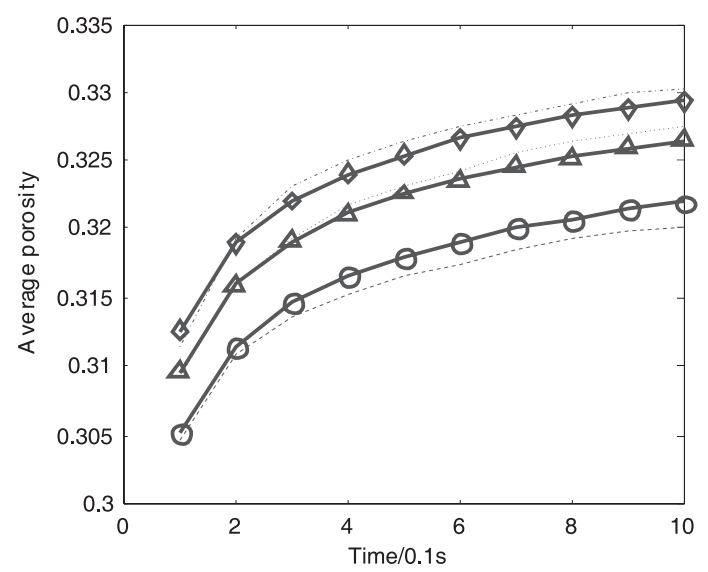

(B) $15.38 \%$ particles fixed

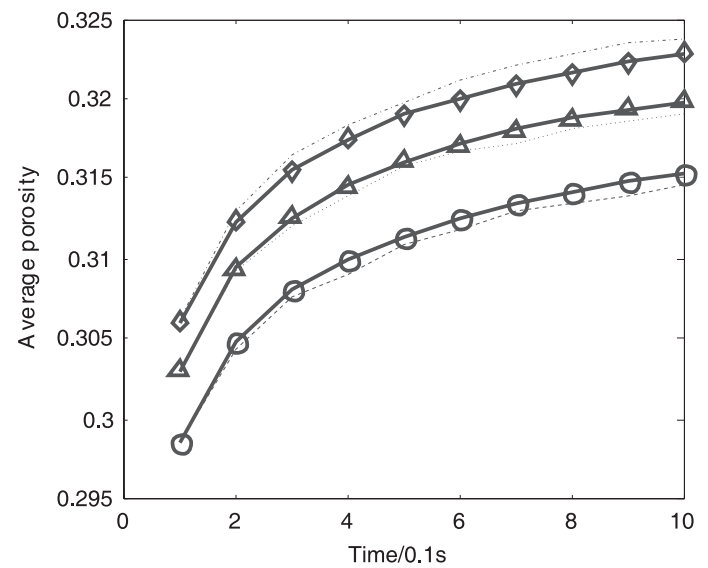

(C) $19.23 \%$ particles fixed

Fig. 6. The porosity evolution of soil mass

(A), (B), and (C) are the porosity evolution of soil mass with the fixed particles of $11.54 \%, 15.38 \%$, and $19.23 \%$ under hydraulic pressure of 10,20 , and $30 \mathrm{KPa}$. shorter the time needed by reaching the maximum level of porosity.

2. During the erosion process, for the soil mass with the same percentage of fixed particles, the porosity under larger hydraulic pressure is larger than that under smaller hydraulic pressure.

3. During the erosion process, under the same hydraulic pressure the more the amount of fixed particles, the smaller the porosity.

Through $\mathrm{A}, \mathrm{B}$, and $\mathrm{C}$ in Fig. 6, we may further fit the curve to obtain the fitting formula of porosity, time, pressure, and fixed particles. The corresponding fitting curve is shown in Fig. 6:

$$
y=\frac{1}{100}\left[2.7 P^{0.16}-3.38 t^{-0.3}-(52 N)^{0.37}+34.01\right]
$$

...where correlation coefficient of fitting is $0.9632 ; y$ is porosity; $P$ is pressure $\mathrm{KPa}$; $t$ is time $0.1 \mathrm{~s}$; and $N$ is the percentage of fixed particles as $0.1154,0.1538$, and 0.1925 .

The equation effectively reflects the above regularities, and the porosity evolution is positively correlated with the hydraulic pressure and time, and negatively correlated with the amount of fixed particles.

\section{Mechanism Interpretation}

The above regularities are interpreted hereby from the aspect of mechanism:

1. Mechanism interpretation of regularity: under hydraulic pressure, non-fixed sediment particles will be scoured and taken away by the water flow. As time extends, more sediment particles will be taken away by the water flow. Hence, with the lengthened time, porosity becomes larger. Meanwhile, fewer particles can be washed away. Thus, the increase range shrank. With the increase of the amount of fixed particles, the amount of sediment particles that can be transferred is reduced and constrained by more fixed sediment particles, thus reaching a larger porosity quickly.

2. Mechanism interpretation of regularity: under the larger hydraulic pressure, more sediment particles in the water may be taken away by water. Hence, under the larger hydraulic pressure the porosity of soil mass will be larger.

3. Mechanism interpretation of regularity: given the substantial amount, fixed particles do not participate in the transport, thus enlarging the group of non-mobile particles; on the other hand, more fixed particles limit the free movement of more non-fixed particles. The two aspects led to smaller soil mass porosity during scouring.

4. Mechanism interpretation of regularity: under hydraulic pressure, free particles are washed away by water flow. The greater the hydraulic pressure, the greater the flow velocity. Thus, more sediment particles will be washed away by water, leading to larger porosity. 

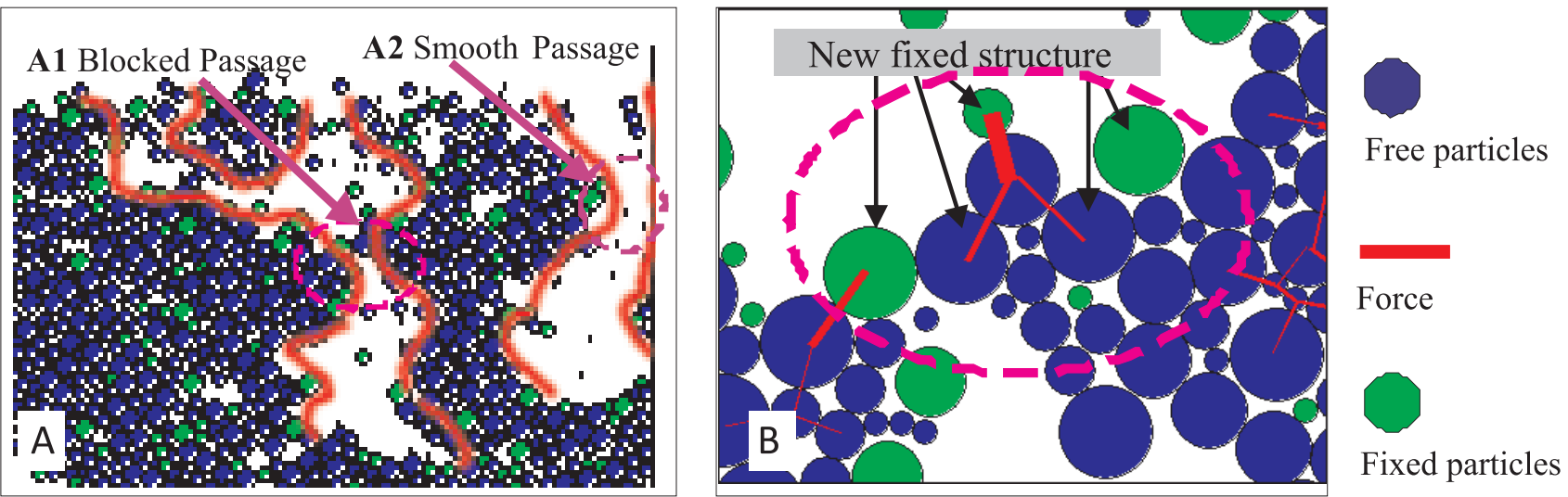

Fig. 7. Blocked/smooth passages and new fixed structure.

\section{Relationship between Fixed Particles and Free Particles}

Our study clarifies the relationship between the fixed particles and free particles. The formation structure of fixed particles contributes to the constraints of the entire soil mass. Under the hydraulic pressure, some free particles move while some others become immobile, which can be called new fixed particles.

These two parts - fixed particles and new fixed particles - constitute the restrained structure of soil mass. And some new fixed structures appear. The new fixed structures show positive impact on the resistance of soil against water scouring.

The more the new fixed particles are, the easily the larger the soil mass of fixed structure can be formed, thereby enhancing the scouring-resistance of the soil body. Free-flowing particles can only move in the newly formed structure, that is, the new passages.

Free particles are transported in free passages formed by fixed particles, and are imposed by the friction of the passage walls. The velocity of a part of free particles is gradually reduced, eventually to 0 . The new fixed structures are formed with fixed particles so that the section of free passages is reduced, ultimately leading to the possible blockage of passages (as shown in A1 of Fig. 7), when the free transport of sediment particles ends. Conversely, some transport passages are dredged, forming smooth passages (as shown in A2 of Fig. 7), which is conducive to the free transport of sediment particles.

Fixed particles are occluded with free particles. Due to the friction and supporting force between the particles, the structure of new fixed particles is formed, as shown in Fig. 7B.

\section{Conclusion}

In this paper, with sediment particles transfer simulation, the following types of phenomena are discovered:

1. The sediment particles in the soil mass are transferred in the role of hydraulic pressure. With the increase in hydraulic pressure and the extension of time, the porosity of soil mass is enlarged and eventually maintains a stable value.

2. Soil mass porosity is reduced as the amount of fixed particles increases.

3. During the erosion process, part of the free particles stopped moving due to the blockage of fixed particles, thereby blocking the passages; a part of the particles is taken away from the soil mass, and the passage is expanded to form smooth passage.

4. Fitting formulas of porosity, time, pressure, and fixed particles of the transfer of sediment particles in the soil.

The mechanism interpretations of these sedimenttransport performances are: under the larger hydraulic pressure, flowing water contains greater kinetic energy, which can take away more sediment particles; fixed particles are occluded with free particles, generating friction among the freely moving sediment particles and hindering the transport of following free particles; under hydraulic pressure, free sediment particles crush each other. The following sediment particles push the sediment particles in the front. If the impetus is larger than resistance, free sediment particles will continue the movement and form smooth passage; conversely, if the impetus is smaller than the resistance, sediment particles will slow down and may be still, forming a blocked passage.

Prospects in this aspect are: under hydraulic pressure, underground sediments in the soil are washed away by water, possibly leading to soil collapse and destruction. A solution is to increase fixed sediment particles and adequately adjust the particle amount in the fixed sediments, thus realizing the control and weakening the underground sediment transport in the soil mass and preventing soil damage. In the actual engineering projects, supports can be set in the soil mass, such as the reinforcing steel bars with large surface friction, sprayed concretes and cement pastes, and sediment particles with large particles and large friction coefficients. Thus, underground sediment transfer can be avoided and the stability of the soil mass can be ensured. 


\section{Acknowledgements}

This work was supported by the National Natural Science Foundation of China (No. 51179177).

\section{References}

1. SUN J.C, WANG G.O., Riverbank Collapse Mechanism Under Scouring. VDM Publishing House Germany, 2010.

2. SUN J. The phenomenon of the floating riverbank collapse by water scouring. International Journal of Ground Sediment and Water. 1 (1), 5, 2014.

3. SUN J. Three Stages Collapse Connection of Riverbank. International Journal of Ground Sediment and Water. 1 (1), $1,2014$.

4. AREKHI S., NIAZI Y., KALTEH A.M. Soil erosion and sediment yield modeling using RS and GIS techniques: a case study, Iran. Arabian Journal of Geosciences, 5 (2), 285, 2012.

5. BISHOP M.A. Aeolian scours as putative signatures of wind erosion and sediment transport direction on Mars. Geomorphology, 125 (4), 569, 2011.

6. WANG G., et al., Underground sand erosion model experimental apparatus. China Patent, 2010.

7. SUN J., WANG G., Transport Model of Underground Sediment in Soils. Scientific World Journal, 1, 2013.

8. WANG G., et al., An underground sand erosion model experimental apparatus and method. China Patent, 2011.

9. SUN J. Mathematical model coupling seepage and sedimentation of solid particles in porous media. Fresenius Environmental Bulletin, 24 (5), 1735, 2015.

10. SUN J. Experimental device and test methods of the interaction wind sand and groundwater. Patent. 2015.

11. SUN J. Simulation test device for the crack block and dredge effect by ground sediment. China, Patent. 2013.

12. TIAN Y.A., et al., Transport of engineered nanoparticles in saturated porous media. Journal of Nanoparticle Research, 12 (7), 2371, 2010.

13. BEN-MOSHE T., DROR I., BERKOWITZ B. Transport of metal oxide nanoparticles in saturated porous media. Chemosphere, 81 (3), 387, 2010.

14. CHOWDHURY I., et al., Mechanisms of $\mathrm{TiO}_{2}$ nanoparticle transport in porous media: Role of solution chemistry, nanoparticle concentration, and flowrate. Journal of Colloid and Interface Science, 360 (2), 548, 2011.

15. SEN T.K., KHILAR K.C. Review on subsurface colloids and colloid-associated contaminant transport in saturated porous media. Advances in Colloid and Interface Science, 119 (2-3), 71, 2006.

16. SEN T.K., MAHAJAN S.P., KHILAR K.C. Colloidassociated contaminant transport in porous media: 1 . Experimental studies. Aiche Journal, 48 (10), 2366, 2002.

17. AUSET M., KELLER A.A. Pore-scale processes that control dispersion of colloids in saturated porous media. Water resources research, 40 (3), 2004.

18. BAEK I.. PITT W.W. Colloid-facilitated radionuclide transport in fractured porous rock. Waste Management, 16 (4), 313, 1996

19. AIREY P.L. Radionuclide Migration around Uranium Ore Bodies in the Alligator Rivers Region of the Northern-Territory of Australia - Analog of Radioactive-Waste Repositories - a Review. Chemical Geology, 55 (3-4), 255, 1986.

20. YAO H.Y., ZHANG Z.H. Numerical Analysis of Saturatedunsaturated Seepage Problem of Anisotropic Permeability Slope under Rainfall Infiltration. Flow in Porous Media
- from Phenomena to Engineering and Beyond, ed. J. Liu, H. Zhang, and X.G. Liu., Marrickville: Orient Acad Forum. 1002, 2009.

21. SUN J.C., WANG G.Q., SUN Q.C. Crack spacing of unsaturated soils in the critical state. Chinese Science Bulletin. 54 (12), 2008, 2009.

22. SUN J.C., GAO Q.C., WANG H.B., LI Y.M. Numerical simulation of coupled rainfall and temperature of unsaturated soils. Key Engineering Materials. 306-308 (2), 1433, 2006.

23. GABET E.J., STERNBERG P. The effects of vegetative ash on infiltration capacity, sediment transport, and the generation of progressively bulked debris flows. Geomorphology, 101 (4), 666, 2008.

24. HAYNES H., VIGNAGA E., HOLMES W.M. Using magnetic resonance imaging for experimental analysis of fine-sediment infiltration into gravel beds. Sedimentology, 56 (7), 1961, 2009.

25. ROBERGE P.R. Polymeric Materials for Underground Piping and Related Systems. Materials Performance, 49 (1), $80,2010$.

26. TERZAGHI K., Der Grundbruch an Stauwerken und seine Verhuetung. Die Wasserkraft, 17, 445, 1922.

27. BAZANT Z. Measuring soil deformation caused by the pressure of the seepage. in $17^{\text {th }}$ International Navigation Congress. Lisbon. 1949.

28. SENTKO M. Der Zeitliche Ablouf des Schwimmsand aufbruches und der Einfluss der geometrischen Anordung der Baugrubenumschliess ungen auf das kritische Gefaelle, in Veroffentlichungen Institu fuer Bodenmechanik. Technische Hochschule: Karsruhe, 1961.

29. BABU G.L.S., VASUDEVAN A.K. Seepage Velocity and Piping Resistance of Coir Fiber Mixed Soils. Journal of irrigation and drainage engineering, 134 (4), 485, 2008.

30. SKEMPTON A.W., BROGAN J.M. Experiments on piping in sandy gravels. Geotechnique, 44 (3), 449, 1994.

31. WAN C.F., FELL R. Laboratory tests on the rate of piping erosion of soils in embankment dams. ASTM geotechnical testing journal, 27 (3), 295, 2004.

32. INDRARATNA B., RADAMPOLA S. Analysis of critical hydraulic gradient for particle movement in filtration. Journal of Geotechnical and Geoenvironmental Engineering, 128 (4), 347, 2002.

33. XIA J.Q., et al., An analysis of soil composition and mechanical properties of riverbanks in a braided reach of the Lower Yellow River. Chinese Science Bulletin, 53 (15), 2400, 2008.

34. YI C., et al., Two-dimensional simulation of underground seepage in a dangerous piping zone of the Jingjiang Great Levee, the middle reach of the Yangtze River. Quarterly Journal of Engineering Geology and Hydrogeology, 40 (1), 85, 2007.

35. SHARMA R.H., KONIETZKY H., KOSUGI K. Numerical analysis of soil pipe effects on hillslope water dynamics. Acta Geotechnica, 5 (1), 33, 2010.

36. BOUILLARD J., LYCZKOWSKI R., GIDASPOW D. Porosity distributions in a fluidized bed with an immersed obstacle. Aiche Journal, 35 (6), 908, 1989.

37. ERGUN S. Fluid flow through packed columns. Chem. Eng. Prog., 48, 1952.

38. CHRISTOPHER. R.H., MIDDLEMAN S. Power-law flow through a packed tube. Industrial \& Engineering Chemistry Fundamentals, 4 (4), 422, 1965.

39. MEYER B.R., BAZAN L.W., WALLS D. Modeling of Proppant Permeability and Inertial Factor for Fluid Flow Through Packed Columns. Effective and Sustainable Hydraulic Fracturing. 2013. 